\title{
Ischemic postconditioning reduces infarct size and microvascular obstruction zone in acute ST-elevation myocardial infarction - a randomized study
}

\author{
Aleksander Araszkiewicz, Marek Grygier, Małgorzta Pyda, Justyna Rajewska, Michał Michalak, \\ Sylwia Sławek-Szmyt, Maciej Lesiak
}

Department of Cardiology, Poznan University of Medical Sciences, Poznan, Poland

Adv Interv Cardiol 2019; 15, 3 (57): 292-300

DOI: https://doi.org/10.5114/aic.2019.87882

\begin{abstract}
A bstract
Introduction: Ischemic postconditiong (postcon) has been reported to reduce infarct size in ST-segment myocardial infarction (STEMI). However, recently a few other studies did not show any effect of postcon or suggested that it may even be harmful.

Aim: We sought to assess whether postcon could reduce infarct size (IS) and the microvascular obstruction (MVO) zone in early presenters with STEMI.

Material and methods: Seventy-four STEMI patients treated with primary coronary intervention $(\mathrm{PCI})<4 \mathrm{~h}$ from symptoms onset were randomly assigned to the postcon group $(n=37)$ or standard PCI group $(n=37)$. Postcon was performed immediately after obtaining reperfusion with 4 balloon occlusions, each lasting $60 \mathrm{~s}$, followed by 60 seconds of reperfusion. Cardiac magnetic resonance was performed in all subjects within 48 to $96 \mathrm{~h}$ after admission. To evaluate the infarct size and MVO, the late gadolinium enhancement (LGE) technique was used. Infarct size was defined as an area greater than $50 \%$ of the maximal signal intensity within LGE. MVO was defined as the area of the absence or hypoenhancement of contrast surrounded by LGE. Infarct size and MVO were determined by planimetry and the summation of discs method.

Results: Postcon was associated with significantly smaller IS (16.42 \pm 9.6 vs. $31.2 \pm 22.2 \mathrm{~g} ; p=0.007)$ and higher ejection fraction ( $59.8 \pm 9.2 \%$ vs. $52.3 \pm 10.2 \%$ ). The extent of MVO was significantly lower in the postcon group in comparison to the control group $(0.76 \pm 1.4$ g vs. $2.2 \pm 3.2 \mathrm{~g} ; p=0.03$ ).

Conclusions: In early presenters with STEMI postcon could significantly reduce infarct size and limit reperfusion injury.

Key words: postconditioning, ST-segment elevation myocardial infarction, microvascular obstruction.

Su m m a ry

Ischemic postconditiong (postcon) has been reported to reduce infarct size in ST-segment myocardial infarction (STEMI). However, recently a few other studies did not show any effect of postcon or suggested that it may even be harmful. The aim of the study was to assess whether postcon could reduce infarct size (IS) and the microvascular obstruction (MVO) zone in early presenters with STEMI. Patients with STEMI treated with primary coronary intervention $(\mathrm{PCI})<4 \mathrm{~h}$ from symptoms onset were randomly assigned to the postcon group or standard PCI group. Postcon was performed immediately after obtaining reperfusion with 4 balloon occlusions, each lasting $60 \mathrm{~s}$, followed by $60 \mathrm{~s}$ of reperfusion. Cardiac magnetic resonance was performed in all subjects within 48 to $96 \mathrm{~h}$ after admission. Postcon was associated with significantly smaller IS and higher ejection fraction. The extent of MVO was significantly lower in the postcon group in comparison to the control group. In early presenters with STEMI postcon could significantly reduce infarct size and limit reperfusion injury.
\end{abstract}

\section{Introduction}

Effective reperfusion therapy has been shown to reduce the myocardial necrosis and significantly improve the prognosis in patients with acute ST-segment ele- vation myocardial infarction (STEMI) [1]. However, restoration of blood flow in the infarct-related artery (IRA) after prolonged occlusion may also have deleterious effects, including extension of the myocardial necrosis

\section{Corresponding author:}

Dr. Aleksander Araszkiewicz, $1^{\text {st }}$ Department of Cardiology, Poznan University of Medical Sciences, 1/2 Długa St, 61-848 Poznan, Poland, phone: +48 608574 375, e-mail: aaraszkiewicz@interia.pl

Received: 24.01.2019, accepted: 14.04.2019. 
zone, the no-reflow phenomenon and reperfusion arrhythmias, making reperfusion therapy a "double-edged sword" [2, 3]. Ischemia-reperfusion injury is a complex phenomenon mediated by several factors, including oxidative stress, intracellular calcium accumulation, rapid restoration of $\mathrm{pH}$, and inflammation, and involves, at least partly, opening of the so-called mitochondrial permeability transition pore (mPTP) [2-8].

Ischemic postconditioning (postcon), defined as repetitive interruptions of the coronary blood flow applied after a period of ischemia, has been thought to inhibit reperfusion injury and has been shown to reduce infarct size in patients with STEMI [9-18]. However, some newer studies did not show any effect of ischemic postcon and even suggested that it may be harmful [19-24].

Microvascular obstruction (MVO) after primary percutaneous coronary intervention ( $\mathrm{PPCl}$ ) for STEMI is a sign of the no-reflow phenomenon in coronary vessels smaller than $200 \mu \mathrm{m}$ despite adequate epicardial reperfusion. MVO is generally thought to be related primarily to reperfusion injury [25]. Cardiovascular magnetic resonance (CMR) provides unique characterization of postinfarctional myocardial injury. The presence of MVO was shown to be associated with larger infarct size, negative left ventricular remodeling and adverse cardiovascular outcomes [26].

Larger infarcts on CMR are consistently associated with larger ventricular volumes, lower ejection fraction and greater MVO, which occurs in $40-60 \%$ of patients treated by primary percutaneous coronary intervention. The influence of postcon on the presence and extent of MVO and its relations with the myocardial necrosis have been studied in only one small clinical trial [27].

\section{Aim}

The present study was designed to test the hypothesis that postconditioning reduces the extent of myocardial necrosis and the MVO zone observed in CMR in highrisk STEMI patients.

\section{Material and methods}

\section{Study design and protocol}

This was a prospective, single-centre, randomized, controlled, open-label study with blinded evaluation. The study was performed according to the provisions of the Declaration of Helsinki. All the participants gave their written informed consent for the investigation. The protocol of the study was approved by the Local Ethics Committee (No. 547/11).

From October 2010 to January 2015 all patients admitted to our department who were eligible for a primary $\mathrm{PCI}$ due to STEMI were considered for enrolment in our study, provided they fulfilled the following inclusion criteria: age $>18$ and $<80$ years old, chest pain $>30$ min and $<6 \mathrm{~h}$ (preferably $<4 \mathrm{~h},<6 \mathrm{~h}$ only if persistent chest pain and ST-segment elevations), ST segment elevation $>0.1 \mathrm{mV}(>0.2 \mathrm{mV}$ in V1-V3) in two contiguous ECG leads, and a thrombolysis in myocardial infarction (TIMI) grade 0 flow in the infarct-related artery. The exclusion criteria were as follows: previous myocardial infarction, previous coronary artery bypass surgery, cardiogenic shock, cardiac arrest, the presence of collateral flow to the infarcted area as evidenced by a Rentrop score $\geq 1$, known renal impairment (serum creatinine $>150 \mathrm{mmol} / \mathrm{l}$ ), persistent atrial fibrillation, ongoing malignant process, a history of gastrointestinal bleeding or stroke, any contraindication for GP IIb/IIla inhibitors, contraindication to cardiac magnetic resonance examination including claustrophobia, implanted electrotherapy devices and any condition that was considered to interfere with the possibility for the patient to complete the study protocol.

All patients received aspirin (300 mg orally), clopidogrel (600 mg orally), and heparin (intravenously at a dose of $70 \mathrm{UI} / \mathrm{kg}$ ) before $\mathrm{PCl}$. Coronary angiography was performed by the percutaneous technique using the transradial approach in most cases and the infarct-related artery was identified. Following angiographic data acquisition, patients were randomized, using a $1: 1$ sequence placed in numbered sealed envelopes, into the control or postcon group. After guidewire placement in the distal part of the vessel, manual thrombectomy was performed in all patients. In the control group a drug-eluting stent was subsequently implanted with direct technique whenever possible. Postconditioning was performed by reinflating the balloon at the same location to a pressure 4-6 atm for $60 \mathrm{~s}$ starting $1 \mathrm{~min}$ after initial reperfusion with thrombectomy. This cycle was then repeated four times. The balloon was located proximally to the site of the index lesion (to avoid embolization with the elements of ruptured plaque). The selection of the inflation-deflation sequence was based on previous experimental and human studies $[8,9]$. Patients in the control group received no additional intervention during $4 \mathrm{~min}$ of reperfusion. The procedure was finished with drug-eluting stent implantation. Ilb/Illa inhibitors (abciximab or eptifibatide) were administered in all patients. All patients were treated with aspirin $75 \mathrm{mg}$ daily lifelong and clopidogrel $75 \mathrm{mg}$ daily for 12 months.

\section{Angiographic analysis}

Angiographic assessment was performed independently by two experienced angiographers who were blinded to each other and to the clinical data. It included TIMI flow grade before and after the procedure, myocardial blush grade (MBG) and Rentrop's score of collateral flow.

\section{CMR image acquisition}

CMR was performed on all the subjects on a $1.5 \mathrm{~T}$ scanner (Siemens Avanto, Erlangen, Germany) 48-96 h 
after admission. Cine imaging with steady-state free precession (SSFP) and late gadolinium enhancement (LGE) imaging were performed in long-axis views and contiguous short-axis slices covering the entire left ventricle (LV). LGE images were acquired 10-15 min after contrast administration using a segmented inversion-recovery gradient-echo sequence.

\section{CMR image analysis}

Analysis was performed offline blinded to patient details using QMass 7.1 (Medis, Leiden, Netherlands) by two experienced observers. LV volumes and function were calculated as previously described. The infarct zone was defined semi-automatically on LGE imaging using the full-width half-maximum (FWHM) technique. LGE areas (infarct size - IS) were defined as areas with a signal intensity of mean $>5$ SD of visually normal, contralateral remote myocardium, as described previously. MVO was defined as areas of hypoenhancement within the infarct zone and was included in the assessed IS. Total MVO volumes and IS volumes were calculated from manual planimetry of short-axis images by the summation of discs and were expressed as IS and MVO volumes and mass, as well as LV myocardial/IS or MVO ratios (Figure 1).

IS and MVO and LV volumes were also indexed by body-surface area.

\section{Study endpoints}

Study end-points were IS expressed as volume and mass, and MVO zone expressed as volume and mass in CMR performed 48-96 $\mathrm{h}$ after intervention.

\section{Statistical analysis}

Calculation of sample size was performed according to the previous studies [27]. Considering an expected reduction of $30 \%$ in the MVO extent with statistical power of $80 \%$ and a probability of type I error of 0.05 with 2-sided test, we calculated a total sample size of 50 patients.
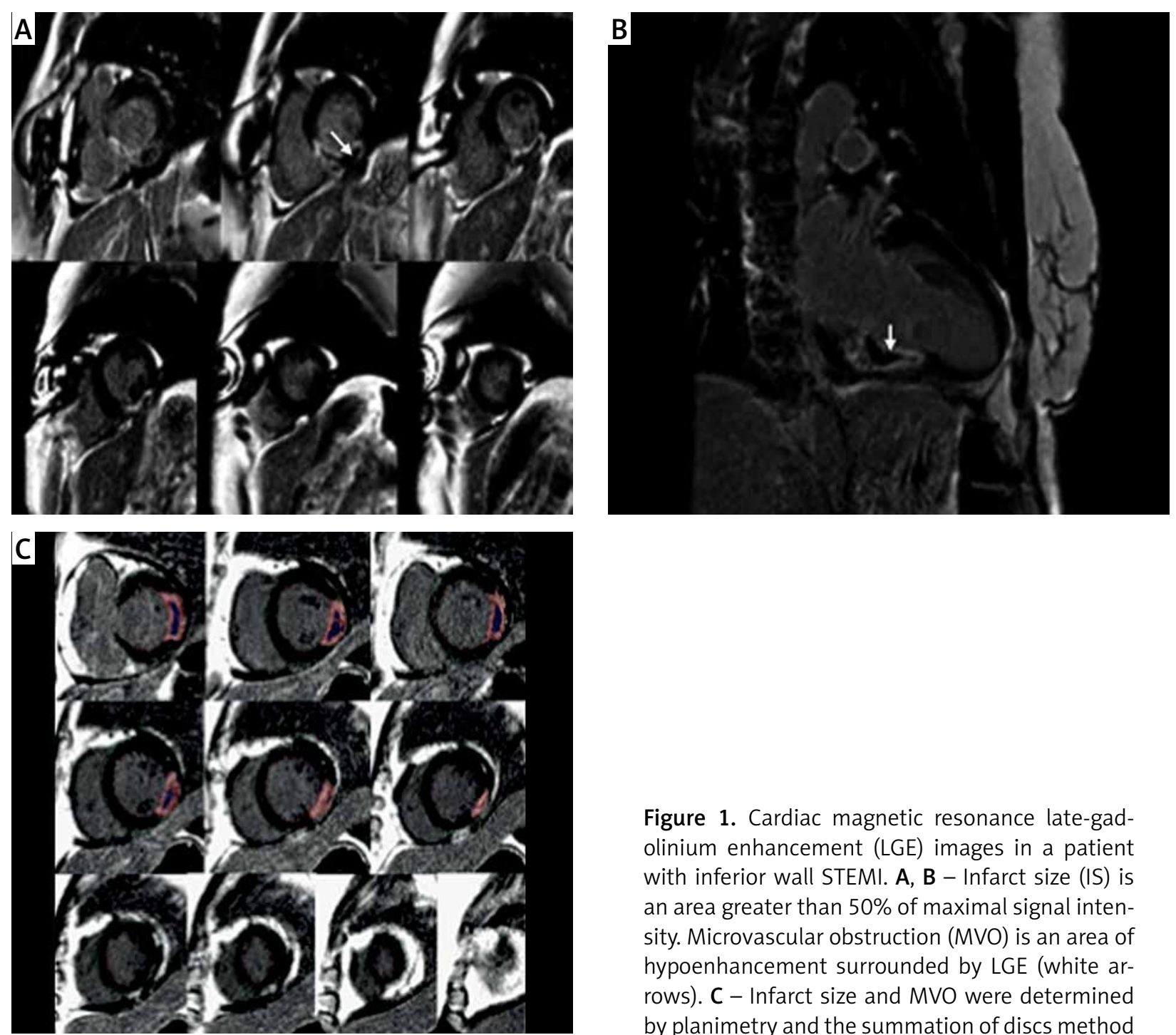

Figure 1. Cardiac magnetic resonance late-gadolinium enhancement (LGE) images in a patient with inferior wall STEMI. A, B - Infarct size (IS) is an area greater than $50 \%$ of maximal signal intensity. Microvascular obstruction (MVO) is an area of hypoenhancement surrounded by LGE (white arrows). C - Infarct size and MVO were determined by planimetry and the summation of discs method 
To compensate for patient dropout, a total of 74 patients were recruited.

Comparisons between groups were performed using unpaired Student's $t$-test or the Mann-Whitney U-test for continuous variables and $\chi^{2}$ or Fisher's exact test for categorical variables. A value of $p<0.05$ was considered statistically significant.

\section{Results}

From a total of 486 screened STEMI patients, 119 patients met the initial criteria and were found initially eligible for the study, but 38 were subsequently excluded as shown in Figure 2. The remaining 81 patients underwent randomization to postcon and control groups, but 7 declined participation, which left 74 patients (37 in the postcon group and 37 in the control group). There were no significant differences between the groups with regard to baseline demographic characteristics (Table I). In postcon patients MBG 3 after PCl was observed significantly more often in comparison to the control group. Angiographic and procedural characteristics are shown in Table II.

Postcon was associated with significantly smaller is (16.6 \pm 9.7 vs. $31.2 \pm 22.9$ g; $p=0.0004)$ and higher left ventricular ejection fraction ( $59.8 \pm 9.2 \%$ vs. $52.3 \pm 10.2 \%$, $p<0.05$ ) (Table II). IS/LV myocardial mass was also sig-

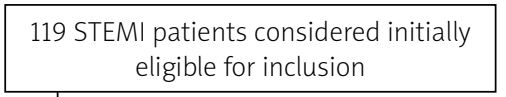

38 patients were excluded:

- 9 refused participation in the trial

- 2 randomised in another scientific study

- 1 patient with Alzheimer disease

- 3 had Rentrop collateral flow grade $>1$

- 18 TIMI flow in IRA > 1

- 2 technical inability to perform postcon

- 2 after pacemaker implantation

- 1 hip prosthesis

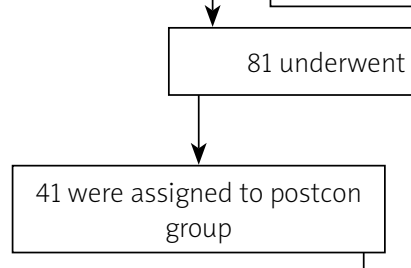

4 patients declined:

- 1 refused further

participation

- 2 poor CMR quality

- 1 claustrophobia

37 were finally analysed

37 were finally analysed

Table I. Clinical characteristics of study groups

\begin{tabular}{|c|c|c|c|}
\hline Characteristics & $\begin{array}{l}\text { Postcon group } \\
(n=37)\end{array}$ & $\begin{array}{l}\text { Control group } \\
\quad(n=37)\end{array}$ & $P$-value \\
\hline Age [years] & $56.9 \pm 10.1(56)$ & $56.7 \pm 9.5$ & 0.7 \\
\hline Male & $26(70.3)$ & $30(81.1)$ & 0.42 \\
\hline Body mass index $\left[\mathrm{kg} / \mathrm{m}^{2}\right]$ & $27.8 \pm 3.9(27.8)$ & $28.1 \pm 4.05(27.7)$ & 0.7 \\
\hline Diabetes mellitus & $6(16.2)$ & $6(16.2)$ & 1.0 \\
\hline Glycemia on admission [mmol/l] & $8.2 \pm 2.5(7.5)$ & $8.7 \pm 4.02(7.4)$ & 0.92 \\
\hline Obesity & $11(30.5)$ & $11(30.5)$ & 1.0 \\
\hline Hypertension & $20(54.1)$ & $20(54.1)$ & 1.0 \\
\hline Family history of angina pectoris & $12(33.3)$ & $9(24.3)$ & 0.44 \\
\hline Smokers & $21(56.8)$ & $18(48.7)$ & 0.32 \\
\hline Symptoms-to balloon time [min] & $210.9 \pm 82.9(186)$ & $216.9 \pm 91.7(194)$ & 0.15 \\
\hline Systolic blood pressure on admission [mm Hg] & $146.7 \pm 21.3$ & $148.8 \pm 18.4$ & 0.2 \\
\hline Diastolic blood pressure on admission [ $\mathrm{mm} \mathrm{Hg}$ ] & $85.4 \pm 10.96$ & $86.5 \pm 11$ & 0.33 \\
\hline Heart rate on admission [beats per minute] & $75.5 \pm 16.3(76)$ & $71.2 \pm 12.7(68)$ & 0.93 \\
\hline Killip-Kimball class 1 on admission & $33(89.2)$ & $31(83.8)$ & 0.3 \\
\hline Multivessel disease & $11(29.7)$ & $17(45.9)$ & 0.18 \\
\hline Anterior myocardial infarction & $11(29.7)$ & $12(32.4)$ & 0.96 \\
\hline Left ventricular ejection fraction on day 3 - echocardiography (\%) & $52.2 \pm 10.8$ & $46.8 \pm 9.2$ & 0.18 \\
\hline
\end{tabular}

The data are expressed as mean \pm standard deviation (SD) or (median) or $n(\%)$. 
Table II. Angiographic characteristics of study group

\begin{tabular}{lccc} 
Characteristics & Postcon group $(n=37)$ & Control group $(n=37)$ & $P$-value \\
\hline Culprit vessel, $n(\%):$ & & & \\
\hline LAD & $11(28.6)$ & $12(32.4)$ & 0.96 \\
\hline RCA & $21(56.7)$ & $19(51.4)$ & 0.62 \\
\hline LCX & $5(13.5)$ & $6(16.2)$ & 0.92 \\
\hline Multivessel disease, $n(\%)$ & $11(29.7)$ & $17(45.9)$ & 0.18 \\
\hline Initial TIMI flow 0, $n$ (\%) & $37(100)$ & $37(100)$ & 1 \\
\hline Final TIMI flow 3, $n$ (\%) & $35(94.6)$ & $32(86.5)$ & 0.84 \\
\hline Final TIMI flow 2, $n$ (\%) & $2(5.4)$ & $5(13.5)$ & 0.2 \\
\hline MBG 3 after PCI, $n$ (\%) & $28(75.7)$ & $18(48.6)$ & 0.02 \\
\hline MBG 0-2 after PCI, $n$ (\%) & $9(24.3)$ & $17(51.4)$ & 0.01 \\
\hline Manual thrombectomy, $n$ (\%) & $37(100)$ & $37(100)$ & 1 \\
\hline TIMI- Thrombolysis in Myocardial Infarction score, MBG - myocardial blush grade, LAD-left anteriordescending artery, LCX-circumflex artery, RCA-right coronary artery.
\end{tabular}

nificantly lower in the postcon group in comparison to the control group (Figure 3).

The extent of MVO was significantly lower in the postcon group in comparison to the control group (0.71 \pm 1.4 g vs. $2.2 \pm 3.2$ g; $p=0.03$ ) (Table II). However, differences in the MVO/LV myocardial mass ratio did not reach statistical significance $(p=0.06)$ (Figure 3$)$.

We observed lower creatinine kinase, and troponin I peak serum levels $(2297 \pm 1391$ vs. $3268 \pm 1163 \mathrm{U} /$; $p<0.001$ and $47.4 \pm 57.1$ vs. $136 \pm 122 \mathrm{ng} / \mathrm{dl} ; p<0.01$ ) in the postcon group compared to the control group (Tables III and IV).

\section{Discussion}

This prospective randomized study evaluating the effect of postconditioning in STEMI patients treated with $\mathrm{PPCl}$ showed that postcon significantly decreased IS and MVO assessed by CMR.

The concept of postconditioning was described for the first time by Zhao et al. [9] in a canine model. The authors documented the effectiveness of intermittent episodes of ischemia and reperfusion applied after prolonged closure of the infarct-related artery. The left anterior descending artery in dogs was ligated for $45 \mathrm{~min}$ and then there were performed intermittent periods of reperfusion and reocclusion $(3 \times 30$ s). Forty-four percent reduction in infarct size as compared with the control group was observed [9].

A few postulated mechanisms of cytoprotective effects of postcon exist. It has been shown that modification of reperfusion affects important mediators of lethal reperfusion injury, reducing oxidative stress, rapid washout of adenosine, osmotic shock and intracellular calcium ion overload [8, 28-30]. Postcon also improves endothelial function, reduces cardiomyocyte apopto- sis, reduces infiltration of neutrophils and, in particular, delays the restoration of neutral $\mathrm{pH}$ [28-31]. Postcon causes an increase in the extracellular space of endogenous ligands, such as adenosine, opioids, bradykinin and PAR-2 agonists (protease activated receptor-2), which, acting on specific membrane receptors, triggers multiple signaling pathways [29-33]. Postcon is a strong impulse, which activates the reperfusion injury salvage kinase (RISK) pathway [32, 33]. This leads consequently to the closure of the key mPTP channels in the mitochondrial membrane [29-33].

Soon after the Zhao et al. publication, the first clinical application of postcon in patients treated with primary percutaneous coronary intervention was made [9]. Studies conducted initially in small groups of patients were promising. Several different algorithms of intermittent reperfusion $-3 \times 30$ s, $3 \times 60$ s, $4 \times 30$ s, $2 \times 90$ s - were applied [11-18]. In small studies of less than 30 patients postcon during primary $\mathrm{PCl}$ seemed to protect the human heart during STEMI, showing a $36 \%$ infarct size reduction as determined by cardiac biomarkers and improved coronary flow reserve and ST-segment resolution [10, 11]. Similarly, Yang et al. reported a $27 \%$ reduction in IS in 41 STEMI patients treated with primary $\mathrm{PCI}$ plus postcon as assessed by scintigraphy [13]. The significant influence of postcon on periprocedural ventricular arrhythmias has also been reported [34]. In a randomized study, Lønborg et al., using magnetic resonance, reported that postcon resulted in a $19 \%$ relative reduction of the infarct size in relation to the area at risk [18]. Recent studies, however, did not confirm the cytoprotective effect of postcon and weakened the enthusiasm for this method. In a few studies it was found that the infarct size did not differ significantly between the two groups, while in two others postcon proved to be even harmful [19-34]. 

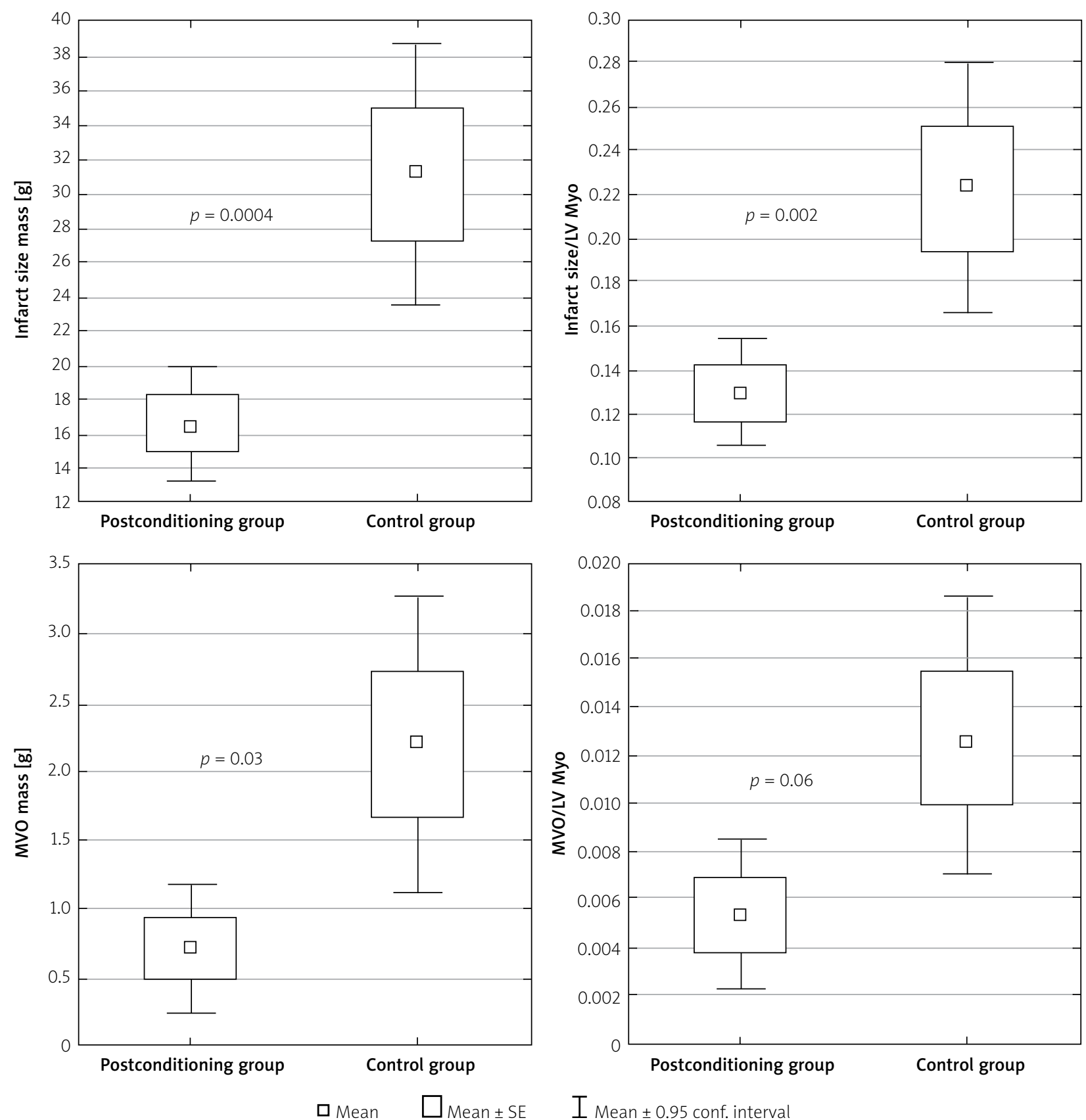

Figure 3. Differences between infarct size (IS) mass, infarct size to left ventricular (LV) myocardial mass index and micro-vessel obstruction (MVO) mass, MVO to LV myocardial mass index

In a trial which included 700 patients, Hahn et al. [19] found that postcon did not improve myocardial reperfusion in patients with STEMI undergoing $\mathrm{PCl}$ with the current standard practice (small size balloon predilatation or thrombus aspiration and stenting). In the biggest randomized trial to date, DANAMI 3-iPOST, Engstrøm et al. found that ischemic postcon in 1234 randomized STEMI patients hospitalized up to $12 \mathrm{~h}$ from symptoms onset failed to reduce the composite outcome of death from any cause and hospitalization for heart failure [20]. Kim et al. [21] in a CMR subanalysis of POST trial showed no benefit of ischemic postcon as an adjunctive treatment of primary $\mathrm{PCl}$. Moreover, Freixa et al. [22] observed in a group of 79 patients that postcon was associated with lower myocardial salvage and lower myocardial salvage index assessed in CMR 1 week after STEMI. They found no significant differences in infarct size and left ventricular ejection fraction between the groups at 1 week and 6 months after MI. The authors suggested that postcon during primary $\mathrm{PCl}$ does not reduce infarct size or improve myocardial function recovery at both short- and long-term follow-up and might have a poten- 
Table III. Parameters of cardiac magnetic resonance in study groups

\begin{tabular}{|c|c|c|c|}
\hline Parameter & Postcon group $(n=37)$ & Control group $(n=37)$ & $P$-value \\
\hline LV EDV [ml] & $132.9 \pm 38.6$ & $163.9 \pm 43.7$ & 0.002 \\
\hline LV SV [ml] & $77.7 \pm 23.2$ & $83.2 \pm 18$ & 0.3 \\
\hline LV EF (\%) & $59.8 \pm 9.2$ & $52.3 \pm 10.2$ & 0.001 \\
\hline LV CO $[1 / \mathrm{min}]$ & $5.6 \pm 1.4$ & $6.2 \pm 1.4$ & 0.08 \\
\hline LV EDV index $\left[\mathrm{ml} / \mathrm{m}^{2}\right]$ & $69.8 \pm 18.8$ & $79.5 \pm 22.7$ & 0.05 \\
\hline LV SV index $\left[\mathrm{ml} / \mathrm{m}^{2}\right]$ & $41.2 \pm 12.8$ & $40.7 \pm 10.5$ & 0.9 \\
\hline $\mathrm{LV} \mathrm{Cl}\left[\mathrm{l} / \mathrm{min} \times \mathrm{m}^{2}\right]$ & $3.1 \pm 0.8$ & $3.1 \pm 0.6$ & 0.8 \\
\hline LV Myo mass index $\left[\mathrm{g} / \mathrm{m}^{2}\right]$ & $65.8 \pm 15.5$ & $69.9 \pm 18.4$ & 0.05 \\
\hline LV Myo volume [ml] & $126.5 \pm 29.3$ & $143.3 \pm 37.5$ & 0.05 \\
\hline LV Myo mass [g] & $125.7 \pm 35.5$ & $143.3 \pm 37.5$ & 0.1 \\
\hline Infarct size - volume [ml] & $16.4 \pm 9.5$ & $29.9 \pm 21.7$ & 0.0006 \\
\hline Infarct size - mass [g] & $16.6 \pm 9.7$ & $31.1 \pm 22.9$ & 0.0004 \\
\hline Infarct size/ LV Myo (\%) & $13 \pm 7$ & $20 \pm 17$ & 0.002 \\
\hline MVO volume [ml] & $0.7 \pm 1.32$ & $2.1 \pm 3.1$ & 0.03 \\
\hline MVO mass [g] & $0.71 \pm 1.4$ & $2.2 \pm 3.2$ & 0.03 \\
\hline MVO/ LV Myo (\%) & $0.5 \pm 0.9$ & $1.3 \pm 0.6$ & 0.06 \\
\hline MVO/infarct size (\%) & $3 \pm 5$ & $5 \pm 19$ & 0.07 \\
\hline
\end{tabular}

Data were presented as mean \pm standard deviation. LV - left ventricular, EDV - end-diastolic volume, SV - stroke volume, EF - ejection fraction, CO - cardiac output, $\mathrm{Cl}$-cardiac index, Myo - left ventricular myocardium, MVO - micro-vessel obstruction.

Table IV. Laboratory markers of myocardial necrosis in postcon group and in control group

\begin{tabular}{lccc} 
Characteristics & Postcon group $(\boldsymbol{n}=\mathbf{3 7})$ & Control group $(\boldsymbol{n}=\mathbf{3 7})$ & $\boldsymbol{P}$-value \\
\hline Creatinine kinase (CK) peak & $2297.24 \pm 1391$ & $3268.9 \pm 1162.9$ & 0.0001 \\
\hline Creatinine kinase MB (CK-MB) peak & $333.6 \pm 232.13$ & $397.4 \pm 175.65$ & 0.01 \\
\hline Troponin I peak & $47.43 \pm 57.115$ & $135.9 \pm 121.9$ & 0.0002
\end{tabular}

tial harmful effect [22]. In the POST-AMI trial, Tarantini et al. randomized 78 patients with STEMI to the postcon or PPCI plus abciximab group [23]. The authors observed that postcon did not have the expected cardioprotective effect and on the contrary it might harm STEMI patients treated with primary $\mathrm{PCl}$ plus abciximab. These contradictory findings may be attributable to differences in $\mathrm{PCl}$ procedures $[24,35]$. Studies in which only a direct stenting technique was allowed or predominantly performed demonstrated the beneficial effect of ischemic postconditioning, whereas studies in which primary $\mathrm{PCl}$ was performed according to the current standard practice such as predilatation with small-sized balloons failed to demonstrate the beneficial effect of postcon [35]. In our study all the patients underwent manual thrombectomy as well as intravenous administration of GP IIb/IIIa inhibitors. Although this type of treatment is no longer used at present, when recruiting patients for the study it seemed to be an optimal reperfusion strategy in combination with primary coronary angioplasty.

One should also note that primary coronary angioplasty of the infarct-related artery can itself become a kind of postconditioning. Pre-dilation cycles, dilatation of the balloon during stent implantation and finally postdilatation of the stent with the non-compliant balloon may act cardioprotectively; hence there is no difference between the patients treated with postcon and the standard primary $\mathrm{PCl}$ group [35].

In the present study a significant reduction in IS (by 47\%) was observed; moreover MVO zone, related primarily to reperfusion injury, was also significantly smaller in comparison to the control group. These observations are in line with earlier clinical experiences looking at the impact of postcon on MVO. Mewton et al. [27] found in 50 STEMI patients that postcon was associated with smaller early and late MVO size. This significant reduction 
was persistent after adjustment for thrombus aspiration, which had no significant effect on either infarct size or on early or late MVO. Attenuation of MVO was associated with infarct size reduction. The authors concluded that mechanical postconditioning significantly reduces MVO in patients with acute STEMI treated with primary angioplasty. In a recent study Traverse et al. observed that postcon had no early beneficial effect on infarct size or left ventricular function compared with routine $\mathrm{PCl}$. However, postconditioning was associated with improved left ventricular remodeling at 1-year follow-up, especially in subjects with the presence of MVO [36].

The protocol of the study may also have a considerable impact on the effectiveness of postcon. Thus, when planning the study we made sure to implement an optimal reperfusion therapy. All our patients received Ilb/ IIla inhibitors and had the infarct-related artery opened using aspiration thrombectomy. Direct stenting was performed whenever possible to avoid microembolisation, which could grossly deteriorate the beneficial effects of postconditioning.

Undoubtedly, there are some limitations of our study. Despite randomization, as one might expect in a relatively small study, there are some imbalances in the baseline characteristics between study groups, albeit not statistical significant (in myocardial mass index and volume). Although MVO extent was significantly lower in comparison to the control group, MVO/LV myocardial mass was not significantly lower. The results should be interpreted with caution.

\section{Conclusions}

Postcon can significantly reduce the infarct size and microvascular obstruction zone in patients treated with primary $\mathrm{PCl}$, although this applies only to early presenters with an extensive area at risk. However, the results should be interpreted with caution and further research is necessary to determine the optimal protocol of the treatment and identify a target group which can best benefit from postconditioning.

\section{Conflict of interest}

The authors declare no conflict of interest.

\section{References}

1. Keelly EC, Boura JA, Grines CL. Primary angioplasty versus intravenous thrombolytic therapy for acute myocardial infaction: a quantitative review of 23 randomised trials. Lancet 2003; 361 : 13-20.

2. Yellon DM, Hausenloy DJ. Myocardial reperfusion injury. N Engl J Med 2007; 357: 1121-35.

3. Braunwald E, Kloner RA. Myocardial reperfusion: a double-edged sword? J Clin Invest 1985; 76: 1713-9.

4. Verma S, Fedak PW, Weisel RD, et al. Fundamentals of reperfusion injury for the clinical cardiologist. Circulation 2002; 105: 2332-6.
5. Matsumura K, Jeremy RW, Schaper J, Becker LC. Progression of myocardial necrosis during reperfusion of ischemic myocardium. Circulation 1998; 97: 795-804.

6. Ito $\mathrm{H}$. No-reflow phenomenon and prognosis in patients with acute myocardial infarction. Nat Clin Pract Cardiovasc Med 2006; 3: 499-506.

7. Kloner RA, Ganote CE, Jennings RB. The no-reflow phenomenon after temporary coronary occlusion in the dog. J Clin Invest 1974; 54: 1496-508.

8. Araszkiewicz A, Grygier M, Lesiak M, Grajek S. The impact of ischemia-reperfusion injury on the effectiveness of primary angioplasty in ST-segment elevation myocardial infarction. Postep Kardiol Inter 2013; 9: 275-81.

9. Zhao ZQ, Corvera JS, Halkos ME, et al. Inhibition of myocardial injury by ischemic postconditioning during reperfusion: comparison with ischemic preconditioning. Am J Physiol Heart Circ Physiol 2003; 285: H579-88.

10. Laskey WK. Brief repetitive balloon occlusions enhance reperfusion during percutaneous coronary intervention for acute myocardial infarction: a pilot study. Catheter Cardiovasc Interv 2005; 65: 361-7.

11. Staat P, Rioufol G, Piot C, et al. Postconditioning the human heart. Circulation 2005; 112: 2143-8.

12. Laskey WK, Yoon S, Calzada N, Ricciardi MJ. Concordant improvements in coronary flow reserve and ST-segment resolution during percutaneous coronary intervention for acute myocardial infarction: a benefit of postconditioning. Catheter Cardiovasc Interv 2008; 72: 212-20.

13. Yang $X C$, Liu $Y$, Wang LF, et al. Reduction in myocardial infarct size by postconditioning in patients after percutaneous coronary intervention. J Invasive Cardiol 2007; 19: 424-30.

14. Thibault H, Piot C, Staat P, et al. Long-term benefit of postconditioning. Circulation 2008; 117: 1037-44.

15. Zhao WS, Xu L, Wang LF, et al. A 60-s postconditioning protocol by percutaneous coronary intervention inhibits myocardial apoptosis in patients with acute myocardial infarction. Apoptosis 2009; 14: 1204-11.

16. Araszkiewicz A, Grygier M, Pyda M, et al. Postconditioning reduces enzymatic infarct size and improves microvascular reperfusion in patients with ST-segment elevation myocardial infarction. Cardiology 2014; 129: 250-7.

17. Sörensson P, Saleh N, Bouvier F, et al. Effect of postconditioning on infarct size in patients with ST elevation myocardial infarction. Heart 2010; 96: 1710-5.

18. Lønborg J, Kelbaek H, Vejlstrup N, et al. Cardioprotective effects of ischemic postconditioning in patients treated with primary percutaneous coronary intervention, evaluated by magnetic resonance. Circ Cardiovasc Interv 2010; 3: 34-41.

19. Hahn JY, Song YB, Kim EK, et al. Ischemic postconditioning during primary percutaneous coronary intervention: the POST randomized trial. Circulation 2013; 128: 1889-96.

20. Engstrøm T, Kelbæk H, Helqvist $\mathrm{S}$, et al. Effect of ischemic postconditioning during primary percutaneous coronary intervention for patients with ST-segment elevation myocardial infarction: a randomized clinical trial. JAMA Cardiol 2017; 2: 490-7.

21. Kim EK, Hahn JY, Song YB, et al. Effect of ischemic postconditioning on myocardial salvage in patients undergoing primary percutaneous coronary intervention for ST-segment elevation myocardial infarction: cardiac magnetic resonance substudy of the POST randomized trial. Int J Cardiovasc Imaging 2015; 31: 629-37. 
22. Freixa X, Bellera N, Ortiz-Perez JT. Ischemic postconditioning revisited: lack of effects on infarct size following primary percutaneous coronary intervention. Eur Heart J 2012; 33: 103-12.

23. Tarantini G, Favaretto E, Perazzolo Marra M, et al. Postconditioning during coronary angioplasty in acute myocardial infarction: the POST-AMI trial. Int J Cardiol 2012; 162: 33-8.

24. Heusch G. Reduction of infarct size by ischemic post-conditioning in humans: fact or fiction? Eur Heart J 2012; 33: 13-5.

25. De Waha S, Desch S, Eitel I Fuernau G, et al. Impact of early vs. late microvascular obstruction assessed by magnetic resonance imaging in long-term outcome after ST-elevation myocardial infarction: a comparison with traditional prognostic markers. Eur Heart J 2010; 31: 2660-8.

26. Wong DTL, Leung MCH, Richardson JD, et al. Cardiac magnetic resonance derived late microvascular obstruction assessment post ST-segment elevation myocardial infarction is the best predictor of left ventricular function: a comparison of angiographic and cardiac magnetic resonance derived measurements. Int J Cardiovasc Imaging 2012; 28: 1971-81.

27. Mewton N, Thibault H, Roubille F, et al. Postconditioning attenuates no-reflow in STEMI patients. Basic Res Cardiol 2013; 108: 383-93.

28. Vinten-Johansen J, Granfeldt A, Mykytenko J, et al. The multidimensional physiological responses to postconditioning. Antioxid Redox Signal 2011; 14: 791-810.

29. Kin $\mathrm{H}$, Zatta AJ, Lofye MT, et al. Postconditioning reduces infarct size via adenosine receptor activation by endogenous adenosine. Cardiovasc Res 2005; 67: 124-33.

30. Ovize M, Baxter GF, Di Lisa F, et al. Postconditioning and protection from reperfusion injury: where do we stand? Position paper from the Working Group of Cellular Biology of the Heart of the European Society of Cardiology. Cardiovasc Res 2010; 87 : 406-23.

31. Ma X, Zhang X, Li C, Luo MJ. Effect of postconditioning on coronary blood flow velocity and endothelial function and $L V$ recovery after myocardial infarction. J Interv Cardiol 2006; 19: 367-75.

32. Hausenloy DJ, Lecour S, Yellon DM. Reperfusion injury salvage kinase and survivor activating factor enhancement prosurvival signaling pathways in ischemic postconditioning: two sides of the same coin. Antioxid Redox Signal 2011; 14: 893-907.

33. Lacerda L, Somers S, Opie LH, Lecour S. Ischaemic postconditioning protects against reperfusion injury via the SAFE pathway. Cardiovasc Res 2009; 84: 201-8.

34. Araszkiewicz A, Grygier M, Pyda M, et al. Postconditioning attenuates early ventricular arrhythmias in patients with high risk ST-segment elevation myocardial infarction. J Cardiol 2015; 65: 459-65.

35. Zhou C, Yao Y, Zheng Z, et al. Stenting technique, gender, and age are associated with cardioprotection by ischaemic postconditioning in primary coronary intervention: a systematic review of 10 randomized trials. Eur Heart J 2012; 33: 3070-7.

36. Traverse JH, Swingen CM, Henry TD, et al. NHLBI-sponsored randomized trial of postconditioning during primary percutaneous coronary intervention for ST-elevation myocardial infarction. Circ Res 2019; 124: 769-78. 